\title{
ESCUTA DO PSIQUIATRA: SINAIS E SINTOMAS DE ANOREXIA NERVOSA E BULIMIA NERVOSA
}

\author{
PSYCHIATRIST'S HEARKEN: SIGNS AND SYMPTOMS OF \\ ANOREXIA NERVOSA AND BULIMIA NERVOSA
}

Ana Teresa F Paccola

Médica Psiquiatra. Grupo de Assistência aos Transtornos Alimentares. Hospital das Clínicas da FMRP- USP

CorReSPondÊnCIA: Rua Altino Arantes, 1900. Jardim América. Ribeirão Preto- SP. e-mail: atfp22@yahoo.com.br

Paccola ATF. Escuta do psiquiatra: sinais e sintomas de anorexia nervosa e bulimia nervosa. Medicina (Ribeirão Preto) 2006; 39 (3): 349-52.

RESUMO: O trabalho que se segue é uma revisão de conceitos psiquiátricos e aspectos clínicos pertinentes para o diagnóstico de anorexia nervosa e bulimia nervosa. Esta revisão foi baseada nos critérios diagnósticos do DSM-IV-TR e do CID-10.

Descritores: Anorexia Nervosa. Bulimia Nervosa. Diagnóstico.

\section{1- APRESENTAÇÃO}

Os transtornos alimentares são, historicamente, temas de interesse constante para a humanidade. $\mathrm{O}$ aumento significativo de pacientes com esse distúrbio nos faz levantar questões a respeito. Como o psiquiatra escuta, vê, sente a anorexia nervosa (AN) e a bulimia nervosa $(\mathrm{BN})$ ? O que se segue é uma revisão sucinta realizada por uma psiquiatra que trabalha, há mais de dois anos, junto ao GRATA-HCFMRP-USP, com foco na Psiquiatria Clínica.

\section{2- ASPECTOS CLÍNICOS DA ANOREXIA NERVOSA}

Anorexia Nervosa (AN) é um transtorno alimentar onde há limitação dietética auto-imposta, padrões bizarros de alimentação, acentuada perda e manutenção do peso abaixo do mínimo considerado normal e um pavor intenso da obesidade ${ }^{1 / 6}$. Manifes- ta-se com maior freqüência em mulheres ( 8 a 10 vezes mais), com início na puberdade ( 0,5 a $1 \%$ das adolescentes). ${ }^{1 / 4} \mathrm{O}$ termo anorexia é incorreto pois se presume que o paciente tenha uma falta de apetite e, exceto nos estágios mais avançados de inanição, existe um rígido controle sobre a fome que por vezes é descrita como monstruosa ${ }^{1,4,5}$. Inicialmente a restrição alimentar se dá em relação a alimentos ricos em carboidratos e lipídios. ${ }^{1,5}$ A refeição, quando é feita, demora excessivamente para se completar, o paciente opta por fazê-las solitariamente e evitam locais públi$\cos .{ }^{1,5}$. Outro comportamento freqüente é a preocupação extrema com a alimentação dos familiares, se esta é completa e nutritiva. ${ }^{5}$ Exercícios físicos extenuantes são comuns ${ }^{1 / 7}$ e cessam somente quando há uma impossibilidade concreta. Alguns se limitam a restringir, apresentando características obsessivoscompulsivas; outros lançam mão de uso de laxantes, diuréticos, anorexígenos e vômitos para compensarem episódio de compulsão alimentar, ou mesmo na au- 
sência de compulsão, apenas para controlar o peso, denotando comportamento impulsivo. ${ }^{1,2,4,7}$ Há sinais físicos característicos de desnutrição (lanugo, emaciamento, p.ex.) e alterações bioquímicas ${ }^{1,2,4,8}$. A AN é a maior causa de morte dentre os transtornos psiquiátricos. ${ }^{1,4,6}$

\section{Quadro I - Critérios diagnósticos para anorexia nervosa, segundo DSM-IV-TR}

A- Recusa em manter peso corporal em um nível igual ou acima do mínimo normal à idade e à altura (índice de massa corporal menor ou igual a $17,5 \mathrm{Kg} / \mathrm{m} 2$ ou $85 \%$ do esperado).

B- Medo intenso de ganhar peso ou de tornar-se gordo, mesmo estando com peso abaixo do normal.

C- Perturbação no modo de vivenciar o peso ou a forma do corpo, influência indevida do peso ou da forma do corpo sobre a auto-avaliação ou negação do baixo peso corporal atual.

D- Nas mulheres pós menarca, amenorréia ( três ciclos menstruais consecutivamente ausentes). Considera-se amenorréia se períodos ocorrem após a administração de hormônio (p.ex. estrógeno).

Especificar se:

- tipo restritivo: sem comportamento compulsivo/ pur-gativo

- tipo compulsão periódica/purgativo: quando há episódio de compulsão alimentar e/ou provocação de vômito, uso de laxantes e/ou diuréticos.

\section{3- ASPECTOS CLÍNICOS DA BULIMIA NER- VOSA}

A BN consiste em repetições de episódios de compulsão alimentar (Binge eating) nos quais a pessoa ingere grandes quantidades de alimento em curto período de tempo com sensação de perda de controle. Além de esforços para controlar o peso com exercício físico excessivo e dietas rigorosas, é utilizada a provocação de vômitos e abuso de diuréticos, laxantes e anorexígenos. ${ }^{1,2,4 / 7} \mathrm{O}$ transtorno é mais comum em mulheres $(9: 1)^{4}$, possui início mais tardio que na
AN e também está mais associado a comportamento impulsivo e a comorbidades. ${ }^{1,2,4,5,8 / 10}$ As pessoas mais acometidas são pessoas ligadas ao mundo da moda, bailarinas, atletas, em países desenvolvidos e classes sociais mais elevadas. ${ }^{1,4}$ Existem estudos em que a prevalência de $\mathrm{BN}$ chega a $40 \%$ das universitárias ${ }^{1}$. Apesar de $70 \%$ dos pacientes possuírem peso normal e os outros $30 \%$ divididos em limite superior e inferior da normalidade ${ }^{4}$, existe também uma preocupação excessiva com o peso e com a forma do corpo ${ }^{1,2,4 / 7}$. Os binge são marcantes e às vezes assustadores e a purgação através do vômito dá grande alívio para a bulímica. ${ }^{1,2,4 / 7}$ Física e bioquimicamente apresentam alterações geralmente pela provocação de vômitos (dentes gastos, hipertrofia de glândulas salivares, ci-

\section{Quadro II- Critérios Diagnósticos para bulimia nervosa, segundo DSM-IV-TR}

A- Episódios recorrentes de compulsão alimentar periódica que é caracterizado por:

(1) Ingestão em período limitado de tempo de uma quantidade de alimentos definitivamente maior do que a maioria das pessoas consumiria em período e circunstância similares.

(2) Sentimento de falta de controle sobre comportamento alimentar durante o episódio (p.ex.: sentimento de incapacidade de parar de comer ou quanto está comendo).

B- Comportamento compensatório inadequado e recorrente, com o fim de prevenir o aumento de peso, como auto-indução de vômitos, uso indevido de laxantes, diuréticos, enemas ou outro medicamento, jejuns ou exercícios excessivos.

C- A compulsão periódica e os comportamentos compensatórios inadequados ocorrem, em média, pelo menos duas vezes por semana, há três meses.

D- Auto-avaliação é indevidamente influenciada pela forma e pelo peso do corpo.

E- O distúrbio não ocorre exclusivamente durante episódios de anorexia.

Especificar se:

- tipo purgativo

- tipo sem purgação 
catrizes no dorso das mãos, hipopotassemia, p. ex) $1,2,4,8$. É comum o surgimento dos dois transtornos alternadamente num mesmo paciente. ${ }^{1,4,7,8,10}$

\section{4- CRITÉRIOS DIAGNÓSTICOS}

Os critérios diagnósticos tais como o DSM (Manual Diagnóstico e Estatístico de Transtornos Mentais da Associação Americana de Psiquiatria) ${ }^{7}$ e o CID (Código Internacional de Doenças da Organização Mundial de Saúde) auxiliam o psiquiatra a nortear seu diagnóstico.

Os transtornos alimentares são alvo de interesse constante e são intensamente estudados pela comunidade médica, há séculos. Contudo, foi no DSMIII, em 1980, que a AN e a BN foram considerados diagnósticos distintos e, pelo CID, somente na décima edição, em 1989 1,4 . Os critérios atuais para diagnóstico de AN e BN, contidos no DSM-IV-TR, estão descritos nos Quadros I e II.

\section{5- DIFICULDADES DIAGNÓSTICAS}

- Diagnóstico Diferencial: todas as possibilidades de doenças sistêmicas devem ser descartadas (p.ex.: tumores, infecções, megaesôfago) $)^{1,2,4,8}$. Alguns transtornos psiquiátricos podem cursar com perda acentuada de peso, como a depressão. ${ }^{1,2,4} \mathrm{O}$ dismorfismo corporal acentuado, o medo de se alimentar ou escolhas bizarras de alimentos ou restrição de certos tipos de alimento sem correspondência com a quantidade de caloria, podem derivar de um quadro delirante, como a esquizofrenia. ${ }^{1,2,4} \mathrm{Os}$ transtornos afetivos podem incluir atos impulsivos/ compulsivos alimentares, e, em particular os que ciclam rapidamente, têm sintomas mistos ou são do tipo II, podem se assemelhar bastante com $\mathrm{BN}^{10}$.
Os transtornos de personalidade, em destaque o borderline, predispõem a comportamento compulsivo alimentar e a outros atos impulsivos, como abuso de drogas, promiscuidade sexual, furtos em lojas, tentativas de suicídio ${ }^{1,2,4,5}$. A avaliação de outros sintomas presentes tende a esclarecer o diagnóstico. Importante frisar que é comum a comorbidade desses e outros transtornos mentais com os transtornos alimentares.

- Dificuldades inerentes ao próprio transtorno alimentar: os sintomas podem ser mascarados pelo paciente, tais como, mentir sobre já ter comido, ocultar o vômito, negar desejo de emagrecer, negar que se vê gordo ${ }^{1,4}$. A família tem dificuldade em enxergar a gravidade da situação; outras vezes o familiar (com freqüência a mãe) acredita que agora está bem melhor (p. ex.: antes ela era gordinha e não arranjava namorado e agora ela é modelo e pode escolher o namorado que desejar...). Os profissionais assistentes devem agir com muito discernimento, se precaver, se questionar e pedir a ajuda de outros profissionais pois podem se envolver e acreditar que o caso também não é tão grave, que o paciente tem plena consciência e vai mudar como prometido, a despeito das evidências clínicas e da história pregressa de insucesso e piora da evolução.

\section{6- CONSIDERAÇÕES FINAIS}

- Observar a PARTE: os transtornos alimentares são regidos por um conjunto de fatores psicológicos, biológicos e ambientais e cada um deve ser identificado e abordado.

- Não perder a dimensão do TODO: a identificação e a abordagem terapêutica devem ter como finalidade a melhor adaptação e o menor sofrimento do paciente e das pessoas que o cercam.

Paccola ATF. Psychiatrist's hearken: signs and symptoms of anorexia nervosa and bulimia nervosa. Medicina (Ribeirão Preto) 2006; 39 (3): 349-52.

ABSTRACT: This work reviews the current psychiatric concepts and the pertinents clinical aspects for the diagnosis of anorexia nervosa and bulimia Nervosa. This review was mainly based on the diagnostics criteria of DSM-IV-TR and ICD-10.

Keywords: Anorexia Nervosa. Bulimia Nervosa. Diagnosis. 


\section{REFERÊNCIAS}

1 - Kaplan HI, Sadock BJ, Grebb JÁ. Compêndio de Psiquiatria. $7^{\text {a }}$ ed. Porto Alegre: Artmed; 1997. p.648-55: Transtornos alimentares.

2 - Nunes Filho P, Bueno JR, Nardi AE . Psiquiatria e saúde mental: conceitos e terapêuticos fundamentais. São Paulo: Editora Atheneu; 1996. p.159-65: Transtornos alimentares.

3 - Grunspun H. Crianças e adolescentes com transtornos psicológicos e do desenvolvimento. São Paulo: Editora Atheneu; 1999. p.131-8: Transtornos alimentares.

4 - Busse SR. Anorexia, bulimia e obesidade. Barueri: Manole; 2004.

5 - Gabbard GO. Psiquiatria psicodinâmica. Baseado no DSM-IV. Porto Alegre: Artmed;1998. p.247-60: Transtornos alimentares.

6 - Dalgalarrondo P. Psicopatologia e semiologia dos transtornos mentais. Porto Alegre: Artes Médicas; 2000. p.209-11: Síndromes referentes ao comportamento alimentar.
7 - American Psychiatric Association. Diagnostic and statistical manual of mental disorders: $4^{\text {th }}$ ed., Washington, DC: APA; 2000.

8 - American Psychiatric Association. Referência rápida às diretrizes para o tratamento de transtornos psiquiátricos. Porto Alegre: Artmed, 2005. p.158-174: Transtornos alimentares.

9 - Tozzi F, Thornton LM, Klump KL, Fichter MM, Halmi KA, Kaplan AS, Strober M,Woodside DB, Crow S, Mitchell J. Rotondo A, Mauri M, Cassano G, Keel P, Plotnicov KH, Pollice C, Lilefeld $\mathrm{LR}$, Berrettini WH, Bulik CM, Kaye WH. Symptom flutuation in eating disorders: correlates of diagnostic crossover. Am J Psychiatry 2005;162 (4): 732-40.

10 - McElrov SL, Kotwal R, Keck PE Jr., Akiskal HS. Comorbity of bipolar and eating disorders: distinct or related disorders with shared disregulations?. J Affect Disord 2005; 86(2/3): 107-27. 